\title{
Wen sollen wir impfen?
}

\author{
Zur Wissenschaft, Ethik, Moral und zu den Folgen \\ der Priorisierung
}

Zusammenfassung: Eine Besonderheit der gegenwärtigen Corona-Pandemie ist die Tatsache, dass die Entwicklung von Impfstoffen noch nie so rasch erfolgte. Ein Jahr nach Beginn und bei noch geringer postinfektiöser Immunität von etwa 1,6 \% der Weltbevölkerung ${ }^{1}$ durchleben wir im Moment die besonders dramatische Situation, dass bereits mehrere Impfstoffe zugelassen sind, die aufgrund der Knappheit ${ }^{2}$ aber nicht allen sofort zur Verfügung stehen können. Die Produktion von $15 \mathrm{Milli}$ arden Impfdosen zur 2-maligen Impfung der Weltbevölkerung baucht Zeit. Es ist vernünftig und in der Medizin üblich, sich die Frage zu stellen, wie mit knappen Ressourcen umgegangen werden soll. Hierbei greifen medizinische, naturwissenschaftliche, moralische und ethische Gesichtspunkte ineinander, was die Diskussion bisweilen erschwert. Der Beitrag befasst sich daher mit der Problematik der Impfpriorisierung in einer allgemeinen interdisziplinären Übersicht und im Epilog mit dem konkreten Anwendungsfall „Deutschland im März 2021“.

Bei weltweit etwa 123 Millionen Infizierten und 2,7 Millionen Toten sowie dem Auftreten neuer gefährlicherer und sich rasch verbreitender Varianten des Virus befinden sich viele Länder mittlerweile (Stand: 21 . März 2021) in der dritten Infektionswelle der Corona-Pandemie. Deren Zentrum bildet mit etwa 45 Millionen Infizierten und

1 Zum Jahreswechsel 2020/2021 lebten 7837693000 Menschen auf der Erde, teilte die Deutsche Stiftung Weltbevölkerung Ende letzten Jahres mit. Teilt man 123 Millionen durch diese Zahl, ergibt sich aufgerundet der angegebene Prozentwert.

2 Hätte die Entwicklung die früher üblichen Zeiten von 5 bis 15 Jahren gedauert, wären die Impfstoffe bei bereits deutlich höherer Immunität nach durchgemachter Infektion auf den Markt gekommen und ihre Knappheit wäre entsprechend deutlich geringer.

- Tab. 1 Übersicht zur Anzahl der Infizierten (in Millionen) und Verstorbenen (in Tausend) weltweit und in verschiedenen Bereichen der Erde. Sowohl das geografische Europa als auch die EU als Teilmenge davon führen das Infektionsgeschehen an (nach Daten der Johns Hopkins University vom 21.3.2021)

\begin{tabular}{|l|l|l|}
\hline Wo & Infizierte (Mio) & Tote (Tsd) \\
\hline Europa* & 44,7 & 883 \\
\hline EU & 31,9 & 592 \\
\hline USA & 29,8 & 542 \\
\hline Brasilien & 11,9 & 290 \\
\hline Indien & 11,6 & 160 \\
\hline Deutschland & 2,7 & 75 \\
\hline weltweit & 123,0 & 2703 \\
\hline
\end{tabular}

*) EU plus Großbritannien, Russland, Ukraine, Belarus, Georgien, Serbien, Moldawien, Kosovo, Bosnien-Herzegowina, Nordmazedonien, Albanien, Montenegro und die Schweiz.

über 880000 Verstorbenen das geografische Europa ( $\triangleright$ Tab. 1).

Bei jeder Pandemie müssen nicht nur die Kranken behandelt, sondern auch die weitere Ausbreitung des Erregers verhindert werden. Hierzu gab es schon vor 102 Jahren während der Influenza-Pandemie die seither bekannten Maßnahmen der Hygiene, der Desinfektion, der körperlichen Distanzierung und viele weitere nicht pharmakologische Interventionen (NPI), die heute mit Anglizismen wie Lockdown und Shutdown bezeichnet werden: Schließungen von Kitas und Schulen, Märkten, Läden, Hotels und Gaststätten, Geschäften, Schwimmbädern und vieler anderer Waren- oder Dienstleistungsgeschäfte bis hin zu Versammlungsverboten, Reise- und Ausgangsbeschränkungen. Mit all diesen Maßnahmen werden Ansteckungen vermieden und damit der R-Wert gesenkt. Liegt er unter 1, dann nimmt die Pandemie ab, liegt er über 1 , dann nimmt sie zu.

Seit gut 3 Monaten sind zudem Impfstoffe gegen den Erreger SARS-CoV-2 bzw. die Erkrankung COVID-19 zugelassen, deren Verfügbarkeit dem weltweiten Bedarf noch längst nicht entspricht. In dieser Situation stellt sich daher zwangsläufig die Frage nach der optimalen Impfstrategie, d.h. danach, wer den Impfstoff bekommen soll, wenn nicht alle kurzfristig geimpft werden können [43]. Wie wir derzeit leidvoll erleben, wird dies noch über Monate bis in den Herbst hinein der Fall sein. Die Antwort auf die Frage lautet daher, dass wir so impfen sollten, dass für alle der größtmögliche Nutzen dabei herauskommt - das ist die Meinung sehr vieler Menschen. Damit stellen sich jedoch 2 Fragen: Was ist der größtmögliche Nutzen und wer sind „alle“?

Die im folgenden Text diskutierten Tatsachen sind nicht neu, sollten jedoch der Diskussion was wir tun sollten, zugrunde gelegt bzw. dabei berücksichtigt werden. Sie betreffen medizinische, empirisch-naturwissenschaftliche aber auch moralische und ethische Gesichtspunkte, die im Folgenden diskutiert werden sollen. Wie sich herausstellen wird, gibt es keine einfache Antwort auf die gestellte Frage. Sie hängt vielmehr von den vorliegenden medizinischen, wissenschaftlichen und moralischen Gegebenheiten selbst ab. Einig ist man sich in der Literatur übrigens sehr weitgehend 
darin, dass zu erst das medizinische Personal geimpft werden muss, um das Gesundheitssystem (und damit auch die Impfungen) am Laufen zu halten. Wenn von Priorisierung die Rede ist, so schreiben beispielsweise die Autoren einer in Science hierzu erschienenen Arbeit, dann geht es eigentlich darum, „wer die Impfdosen bekommt, die danach folgend verimpft werden"3 [38].

\section{Tote vermeiden}

Dass man knappe Ressourcen in der Medizin so verteilen sollte, dass möglichst wenige Menschen sterben, leuchtet jedem unmittelbar ein. Es sollten daher diejenigen geimpft werden, die am stärksten gefährdet sind (Menschen über 80 Jahre und Menschen in Pflegeheimen sowie deren Pflegekräfte), damit die Zahl der Toten minimiert wird. In Deutschland hat man noch den Gesichtspunkt berücksichtigt, dass die Intensivstationen nicht überlastet werden sollen, damit die Gesundheitsfürsorge für alle Menschen (egal, woran sie erkrankt sind) zu jedem Zeitpunkt gewährleistet ist. Das klingt zunächst sehr plausibel. „Alle“ sind damit wirklich alle (nicht nur die Corona-Infizierten), und „Tote vermeiden“ muss in der Medizin oberstes Gebot sein. - Wirklich?

Mitarbeiter der in Pflegeheimen im Einsatz befindlichen mobilen Impfteams berichten immer wieder davon, dass sie die notwendige zweite Impfung 3 Wochen nach der ersten nicht durchführen können, weil die Betroffenen mittlerweile verstorben sind - weder an Corona noch an einer Impfreaktion, sondern weil sie sehr alt und krank (und deswegen im Pflegeheim) waren. Die Impfung hatte damit keinen Effekt. Diese Beobachtung stimmt nachdenklich, denn wenn Ressourcen knapp

3 Weil sich dies gerade in Baden-Württemberg (Sozialministerium) noch nicht herumgesprochen hat, sei der ganze Satz vollständig im original zitiert: „Although the earliest doses of vaccines will be given to front-line health care workers under plans such as those from the COVAX initiative and the U.S. National Academies of Sciences, Engineering, and Medicine (NASEM) recommendations, our work is focused on informing the prioritization of the doses that follow."

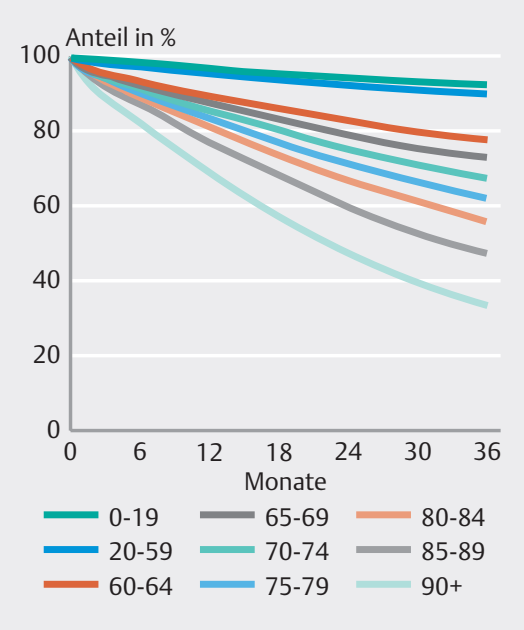

- Abb. 1 Durchschnittliche Verweildauer in der Pflegebedürftigkeit (in Monaten) bei allen Pflegebedürftigen in Abhängigkeit von deren Alter (zusammengefasst in Altersgruppen) (nach Daten aus [22])

sind, dann sollten sie so effizient wie möglich genutzt werden.

\section{Jahre gewinnen}

Es ist in der Medizin durchaus üblich, bei knappen Ressourcen das Alter der möglichen Empfänger bei der Allokation in Betracht zu ziehen. Warten beispielsweise ein 20-Jähriger und ein 80-Jähriger auf ein Spenderorgan und sind die Fälle bis auf das Alter medizinisch nicht unterschiedlich gelagert, wird der 20-Jährige das Organ erhalten. Das Ziel medizinischen Handelns, so lässt sich allgemein festhalten, besteht nicht in der Minimierung der Anzahl der Toten, sondern in der Maximierung der Jahre von gelebtem Leben. Nicht die Zahl der Toten, sondern die Zahl der verlorenen Jahre (Years of Lives Lost; YLL) soll minimiert werden. Darüber ist man sich international einig. Wenn dem aber so ist, dann könnte es problematisch sein, beim Impfen mit den Ältesten zu beginnen. Denn von den erstmals Pflegebedürftigen ist fast jeder Vierte $(23,7 \%)$ bereits nach einem halben Jahr und knapp jeder Dritte (31,9\%) nach einem Jahr verstorben. Nach 3 Jahren ist über die Hälfte $(51,4 \%)$ verstorben. Im Hinblick auf die gerechte Verteilung knapper Güter in der Medizin haben sich hierzu auch schon andere Wissenschaftler Gedanken gemacht $[5,17]$. Betrachtet man alle Pflegebedürftigen, so ist die Überlebenszeit im Heim

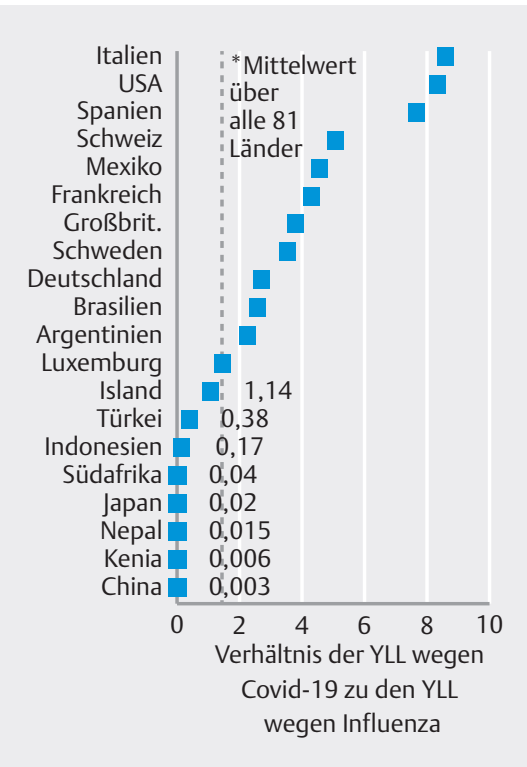

- Abb. 2 Verhältnis der YLL durch COVID-19 zu den YLL durch Influenza (Durchschnitt aus den Jahren davor) für ausgewählte Länder (nach Daten aus [3]).

sehr klar altersabhängig ( $>$ Abb. 1). Geht man von einer durchschnittlichen Verweildauer aller Pflegebedürftigen im Heim von etwa 3 Jahren ${ }^{4}$ aus, ist zumindest denkbar, dass die Priorisierung nach Alter nicht dazu führt, dass die Lebenszeit aller maximiert wird. Dies ist zumindest das Ergebnis einer weiteren Studie aus Großbritannien, die den durch COVID-19 verursachten YLL in 81 Ländern untersuchte [3]. Unter Heranziehung entsprechender großer Datenbanken verzeichneten die Autoren (mit Stand vom 6. Januar 2021) 1279866 Tote und insgesamt 20507518 verlorene Lebensjahre aufgrund von COVID-19. Das ergibt rechnerisch für jeden einzelnen Corona-Toten einen Verlust an Lebenszeit von etwa 16 Jahren.

Halten wir kurz bei dieser methodisch sehr aufwändigen Studie inne. Um das genannte Ergebnis - über 20 Millionen (!) YLL durch Corona - besser einordnen zu können, verglichen die Autoren diese mit den YLL aufgrund anderer Todesursachen ( $\triangleright$ Abb. 2). Bestimmt man die YLL pro 100000 Einwohner für die hochentwickelten Länder,

4 Die Tendenz dieser Zahl ist in den letzten Jahren eher abnehmend. Sie ist länderspezifisch und liegt z.B. in Großbritannien bei nur gut 2 Jahren [9]. 


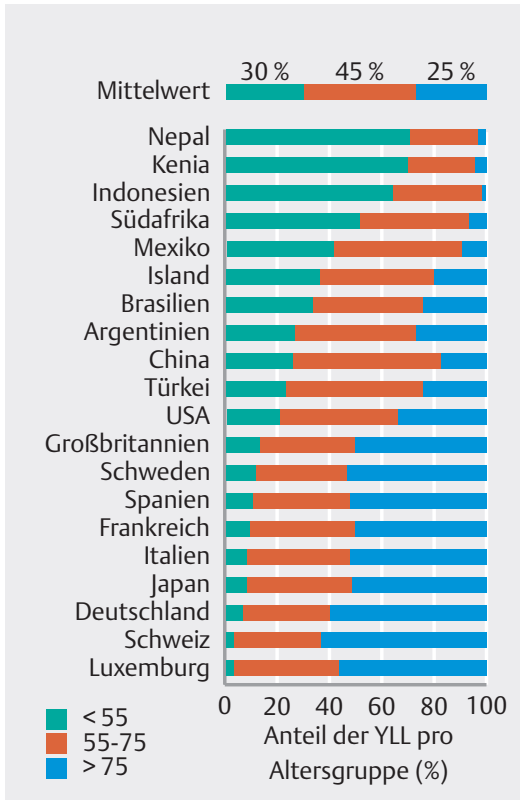

- Abb. 3 Prozentualer Anteil dreier Altersgruppen (unter 55, 55-75, über 75 Jahre) an den YLL in ausgewählten Ländern (nach Daten aus [3]). Die Mittelwerte beziehen sich auf alle 79 in der Originalabbildung aufgeführten Länder.

so liegt die Zahl für COVID-19 bei dem 2bis 9-Fachen der YLL durch Influenza, mehr als dem 2- bis 8-Fachen der YLL durch Verkehrsunfälle und bei etwa einem Viertel bis der Hälfte der YLL durch kardiovaskuläre Erkrankungen, der vielerorts häufigsten Todesursache [3]. Durch den wöchentlichen Vergleich der Corona-Mortalität mit der Übersterblichkeit in einigen der untersuchten Länder (es war nicht überall möglich) ergab sich ferner, dass die Corona-Mortalität deutlich unterschätzt wurde und die YLL durch Corona wahrscheinlich um etwa den Faktor 3 höher liegen. ${ }^{5}$

Die Autoren berechneten weiterhin die YLL nach Altersgruppen. Über alle Länder hinweg trugen die über 75-Jährigen $25 \%$ zu den durch Corona verlorenen Lebensjahren bei, die 55 - bis 75 -Jährigen $45 \%$ und die unter 75 -Jährigen $30 \%$. Zwischen den einzelnen Ländern fanden die Autoren erheb-

5 Die Variabilität zwischen einzelnen Ländern erwies sich als erheblich: In Belgien stimmte die Corona-Mortalität mit der Übersterblichkeit recht gut überein, ,wohingegen für Kroatien, Griechenland und Südkorea die Analyse der Übersterblichkeit nahelegt, dass wir die YLL um einen Faktor von mehr als 12 unterschätzen" [3]. liche Unterschiede ( $\triangleright$ Abb. $\mathbf{3}$ ). So liegt in Nepal, Kenia und Togo der Anteil der Menschen unter 55 Jahren an den YLL bei über $70 \%$ und der der Menschen über 75 Jahren bei unter $5 \%$. In Deutschland und den meisten Nachbarländern tragen hingegen die über 75-Jährigen mit $50 \%$ oder mehr (Deutschland: über 60\%) die Hauptlast der YLL, wohingegen der Anteil unter 75-Jährigen bei unter $10 \%$ (Deutschland: $7 \%$ ) der verlorenen Lebensjahre liegt. „In Ländern mit höherem Einkommen wird ein größerer Anteil der YLL von der ältesten Gruppe getragen als von den jüngsten Altersgruppen. Das entgegengesetzte Muster zeigt sich in Ländern mit niedrigem und mittlerem Einkommen, wo ein großer Anteil der YLL von Personen getragen wird, die im Alter von 55 Jahren oder jünger sterben “ 6 , kommentieren die Autoren das recht deutliche Muster, das sich in ihren Daten zeigt [3].

Halten wir fest: Im Hinblick auf Deutschland ergibt sich aus diesen Daten die Erkenntnis, dass auch bei Zugrundelegung der YLL (und nicht der Anzahl der Verstorbenen) die Impfstrategie zu rechtfertigen ist, die derzeit hierzulande und auch beispielsweise in Großbritannien implementiert ist [18, 30]. Für Entwicklungsländer und möglicherweise manche Schwellenländer sähe eine optimale Impfstrategie anders aus [3, 9].

Stellen Sie sich 2 Krebstherapien vor, die beide das Leben der behandelten Patienten im Mittel um 10 Jahre verlängern. Die eine hat Nebenwirkungen, die andere nicht. Bewertet man diese Therapien im Hinblick darauf, wie viele Tote vermieden werden oder wie viele Lebensjahre nicht verloren gehen, erscheinen sie gleichwertig. Bis in die 1960er-Jahre reichen daher die Bemühungen, auch die Lebensqualität in die Bewertung medizinischer Maßnahmen mit einzubeziehen. Die von Gesundheitsökonomen hierzu eingeführte und auf der Welt vielfach praktizierte Lösung des Problems hat den Namen „Quality Adjusted Life Year“, zu Deutsch „qualitätskorri-

6 „In higher income countries, a larger proportion of the YLL is borne by the oldest group compared to the youngest age groups. The opposite pattern appears in low and mid-income countries, where a large fraction of the YLL are from individuals dying at ages 55 or younger." giertes Lebensjahr“, abgekürzt QALY. In das QALY gehen sowohl die Verlängerung der Lebenszeit als auch die Lebensqualität ein [16]. In den 1990er-Jahren wurde dann das DALY („Disability Adjusted Life Year“) eingeführt, das auf ähnlichen Gedanken beruht [7, 36, 37]. Weder QALYs noch DALYs wurden jedoch im Zusammenhang mit der Corona-Pandemie diskutiert. Letztlich lag das daran, dass die Bedrohung zu groß und die Informationen über das Virus zu gering waren, um diese Größen zu berechnen [ 9 , 11]. Moore und Mitarbeiter [18] formulieren dies wie folgt: „Das noch nie dagewesene Ausmaß der Pandemie setzt die üblichen Maßstäbe [des] gesundheitsökonomischen Ansatzes außer Kraft"7. Das gibt zu denken, denn die westliche Welt verlangt solche Berechnungen von den Entwicklungs- oder Schwellenländern vor nahezu jeglicher gesundheitsbezogener finanzieller Hilfe; nur wenn wir selbst betroffen sind, dann setzen wir dies aus.

\section{Minimierung der Infektionen}

Neben der Verhinderung der direkten Ansteckung der am stärksten durch die Krankheit Gefährdeten (also beispielsweise der Menschen in hohem Alter), kann man auch versuchen, diejenigen zu impfen, welche die meisten anderen Menschen anstecken können (also eher die mobileren, sozial aktiveren jüngeren Menschen). Unter bestimmten Voraussetzungen kann dieses Prinzip der Minimierung von Ansteckung sogar die für alle Beteiligten bessere Strategie sein. Welche sind das?

Eine im Fachblatt Science publizierte Arbeit [38] geht anhand von Modellrechnungen zum weiteren Verlauf der Pandemie unter bestimmten Annahmen bezüglich dreier Varianzquellen diesem Problem auf den Grund: „Erstens haben wir Varianz in der Leistungsfähigkeit des Impfstoffs berücksichtigt, einschließlich seiner Gesamtwirksamkeit, einer hypothetischen Abnahme der Wirksamkeit mit zunehmendem Alter und der Fähigkeit des Impfstoffs, die Ansteckung zu blockieren. Zweitens haben wir die Abhängigkeit sowohl der Anfälligkeit für

7 „[...] the unprecedented scale of the pandemic invalidates the usual metrics [of the] health-economic approach.“ 
Infektionen als auch der Sterblichkeit vom Alter berücksichtigt. Drittens haben wir Varianz im Verhalten der Bevölkerung und der Maßnahmen berücksichtigt - einschließlich der Altersverteilung, der altersabhängigen Kontaktraten und des Anteils seropositiver Personen nach Alter - sowie die Geschwindigkeit und den Zeitpunkt der Einführung des Impfstoffs im Verhältnis zum Infektionsgeschehen " 8 [38]. Bereits vor mehr als einem Jahrzehnt wurde beispielsweise beobachtet, dass im Falle einer Influenza-Pandemie eine optimale Impfung durch die Priorisierung von Schulkindern und Erwachsenen im Alter von 30 bis 39 Jahren erreicht wird. „Schulkinder sind für die meisten Ansteckungen verantwortlich, und ihre Eltern dienen als Brücke zur übrigen Bevölkerung. Unsere Ergebnisse deuten darauf hin, dass die Berücksichtigung der altersspezifischen Übertragungsdynamik für die optimale Allokation von Influenza-Impfstoffen von größter Bedeutung ist“, schrieben die Autoren [39] im Fachblatt Science. Die Vorgaben der US-Regierung zur Impfpriorisierung wurden daraufhin entsprechend geändert.

Die Ergebnisse von Bubar und Mitarbeitern zu einer optimalen Priorisierung bei Impfungen gegen COVID-19 lassen sich wie folgt zusammenfassen: Für Impfstoffe mit 90\%ige Wirksamkeit gilt, dass bei einem R-Wert von >1,3 die Priorisierung von Menschen über 60 Jahren die Sterblichkeit und die YLL am besten mindern. Bei einem R-Wert von $<1,2$ hingegen war diesbezüglich die Priorisierung von Menschen im Alter von 20 bis 40 Jahren am günstigsten. „Die Priorisierung der 20- bis 49-Jährigen minimiert dagegen die Zahl der Neuinfizierten bei allen untersuchten R-Werten zwischen 1,1 und 2,0“ [38]. Um die Auswirkungen nationaler Unterschiede zu untersuchen, wurden die Modell-

8 "First, we considered variation in the performance of the vaccine, including its overall efficacy, a hypothetical decrease in efficacy by age, and the vaccine's ability to block transmission. Second, we considered variation in both susceptibility to infection and the infection fatality rate by age. Third, we considered variation in the population and policy-including the age distribution, age-stratified contact rates, and initial fraction of seropositive individuals by age-and the speed and timing of the vaccine's rollout relative to transmission." rechnungen für Demografie und Struktur der Kontakte für die Länder USA, Belgien, Brasilien, China, Indien, Polen, Südafrika, Spanien und Zimbabwe durchgeführt. Bei einer hohen Infektionsdynamik $(R=1,5)$ vermindert das Impfen der über 60-Jährigen die Mortalität in allen Ländern am besten - unabhängig von der Geschwindigkeit des Impfens (gemessen als Prozentsatz der Bevölkerung, der pro Tag geimpft wird; verglichen wurden die Werte $0,1 \%$ [niedrig] und 0,2\% [hoch]). Bei einer niedrigeren Infektionsdynamik $(R=1,15)$ hingegen hängt es von der Geschwindigkeit des Impfens und der Menge von Impfdosen (bezogen auf die Zahl der Bevölkerung) ab, welches Alter man priorisieren sollte: In Südafrika, Brasilien, Zimbabwe und Indien wird die Mortalität am besten durch Impfungen der über 60-Jährigen reduziert. In Polen, China, Spanien, den USA und Belgien hingegen reduziert das Impfen der Menschen im Alter zwischen 20 und 49 Jahren die Sterblichkeit am besten $[38]^{9}$. Will man das Infektionsgeschehen mit höchster Priorität in den Griff bekommen, so ist das Impfen der Älteren meist nicht die beste Strategie - auch dies zeigen die Modellrechnungen.

Insgesamt zeigt diese Studie, dass es keine einheitliche Lösung für die Priorisierung über verschiedene Länder bzw. über die gesamte Landschaft der untersuchten Parameter hinweg gibt. „Wenn es große Unterschiede im Sterberisiko mit dem Alter gibt, oder wenn die Reproduktionszahl $\mathrm{R}_{0}$ hoch ist, werden ältere Menschen eher für die Impfung priorisiert. Im Gegensatz dazu können eine hohe Impfstoffwirksamkeit, eine höhere Zahl an Sozialkontakten bei jüngeren Menschen und das breite Einhalten von NPI die Priorisierung in Richtung

9 Zwar wurde in Dänemark eine deutliche Schutzwirkung einer vergangenen Infektion (in der ersten Welle) gegenüber einer Neuinfektion (in der zweiten Welle) gefunden [42], die vielen Varianten scheinen sich jedoch wenig an vorhandenen Antikörpern gegen den Wildtyp zu stören. Vor dem Hintergrund dieser Erfahrungen aus Manaus, Brasilien (auch ein hoher Anteil an seropositiven Personen verhinderte nicht die Zahl der Neuinfektionen mit neuen Varianten; [40]) werden die günstigen Auswirkungen der Berücksichtigung von Seropositivität beim Impfen hier nicht weiter diskutiert. jüngerer Altersgruppen verschieben “10, schreiben die Autoren eines Kommentars [45]. Es macht also Sinn, eine Impfstrategie nicht starr zu verfolgen, sondern sie den Gegebenheiten des Infektionsgeschehens hier und jetzt anzupassen.

\section{Zweckrationale Werte}

Menschen mögen es im Allgemeinen nicht, wenn sie als „Mittel zum Zweck“ betrachtet werden ${ }^{11}$. In der bisherigen Diskussion ging es daher auch zunächst um intrinsische Werte, wie das Vermeiden von Todesfällen oder das Leben selbst. Diese Werte sind nicht für irgendetwas anderes gut (also Mittel zum Zweck), sondern selber „Endzweck“, wie man auch sagt. Bei zweckrationalen Werten hingegen geht es darum, dass jemand oder etwas einen Wert hat, um etwas anderes bei einem anderen zu bewirken. So sind sich z. B. viele Menschen darin einig, dass Mütter bevorzugt behandelt werden sollen - ganz einfach, weil die Kleinen sie brauchen. ${ }^{12}$ Auch in der Medizin spielen zweckrationale Werte bei der Allokation knapper Ressourcen eine Rolle. Beispielsweise leuchtet jedem der Gedanke ein, die im System arbeitenden Menschen besonders zu schützen, weil das System ansonsten als Ganzes in seiner Funktion gefährdet ist. Denn ein Zusammenbruch der medizinischen Versorgung aufgrund von Personalmangel würde nicht nur den COVID-19-Kranken schaden, sondern alle kranken Menschen betreffen. Auch die Priorisierung des medizinischen Personals beim Impfen ist daher weitgehend unumstritten, zumal sie je nach Studie auch ein 3- bis 10fach höheres Erkrankungsrisiko haben. Bei den Impfungen gegen Influenza wird häufig - um ein weiteres Beispiel für zweckra-

10 "When there are stark differences in mortality risk with age, or when the reproductive number $R_{0}$ is high, older people are more likely to be prioritized for vaccination. By contrast, high vaccine efficacy, the amount of social contact among younger people, and widespread adherence to nonpharmaceutical interventions can shift prioritization toward younger ages."

11 Sie können sich dabei auf Gedanken der Aufklärung, wie sie z.B. Immanuel Kant formuliert hat, berufen.

12 Nicht umsonst heißt es seit Jahrhunderten bei Seenot: Frauen und Kinder zuerst in die Rettungsboote. 
tionale Werte anzuführen - der Gedanke, Kinder zu impfen, um ältere Menschen zu schützen, vielerorts in die Praxis umgesetzt $[4,10]$.

Hierzulande sind zweckrationale Werte heftig diskutiert, wenn es um die Frage geht, ob Erzieherinnen und Lehrer beim Impfen besondere Berücksichtigung finden sollen. Bildung ist für die Existenz unserer Gesellschaft notwendig, der Schutz der dafür Zuständigen beim Öffnen der Kitas und SchuIen nach wochenlangen Schließungen daher nicht von der Hand zu weisen. Die Kosten von weniger Bildung sind enorm; die ökonomischen lassen sich berechnen (es geht um Billionen [28]), die psychologischen und gesellschaftlichen (noch) nicht [24].

Halten wir fest: Das Heranziehen zweckrationaler Werte ist in der Medizin durchaus üblich. Wie weit hierbei nur Menschen im Bereich der Medizin oder Menschen in weiteren „systemrelevanten“ (gemeint ist unser Gesellschaftssystem) Bereichen wie Bildung, Versorgung mit Nahrungsmitteln, innere und äußere Sicherheit oder kritische Infrastruktur ins moralische Kalkül einbezogen werden, ist eine der vielen hier aufgeworfenen ethischen Fragen, die anhand wissenschaftlicher Fakten und moralischer Grundsätze zu beantworten sind.

\section{Moralische Dilemmata}

Unsere moralischen Grundsätze können dabei durchaus auch Gegenstand empirischer Forschung sein. Ein bekanntes Beispiel ist das internationale Moral Machine Experiment, bei dem 40 Millionen in 10 Sprachen dargebotene moralische Entscheidungen, die autonome Fahrzeuge zur Minimierung des Schadens bei Unfällen treffen müssen, von Millionen von Menschen aus 233 Ländern automatisch über das Internet abgefragt wurden. Die globalen moralischen Präferenzen beinhalten, dass man lieber handelt als nicht handelt, Menschen mit höherem sozialen Status gegenüber niederem sozialen Status bevorzugt, nicht kriminelle Menschen gegenüber kriminellen, größere Menschen gegenüber kleineren, Mädchen gegenüber Jungen und jüngere Menschen gegenüber älteren. Auch sind die meisten Menschen dafür, dass grundsätzlich so wenig wie

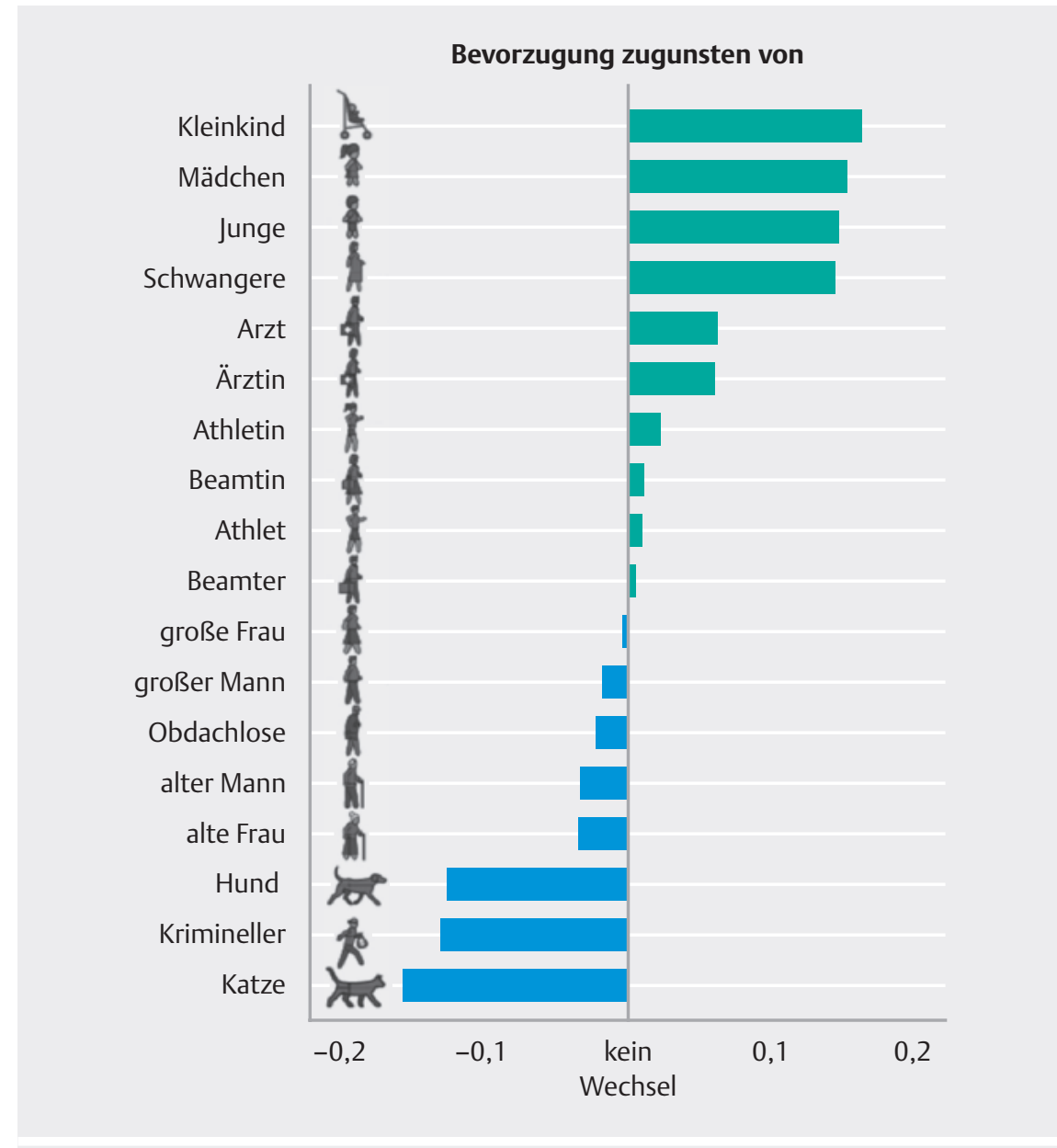

- Abb. 4 Ergebnisse des Moral Machine Experiments, d. h. Mittelwerte aus 40 Millionen moralischen Entscheidungen weltweit. Die grünen Balken nach rechts zeigen an, um wie viel die Wahrscheinlichkeit, Schaden abzuwenden, in Abhängigkeit vom links genannten „Subjekt des Schadens “ zunimmt, die blauen Balken nach links zeigen dagegen eine Abnahme dieser Wahrscheinlichkeit (nach Daten aus [31])

möglich Menschen Schaden nehmen sollten ( $\triangleright$ Abb. 4).

Zudem gab es individuelle Variationen in den Präferenzen, die auf den demografischen Merkmalen der Befragten basierten. Außerdem ergaben sich kulturübergreifende ethische Unterschiede und es wurden „3 große Ländercluster aufgedeckt, [...] die mit modernen Institutionen und tiefen kulturellen Merkmalen korrelieren “ [31]. Interessant ist beispielsweise, dass in Südamerika junge Menschen gegenüber älteren sehr deutlich priorisiert werden, wohingegen dies in Südostasien genau umgekehrt ist. Speziell im Hinblick auf knappe Ressourcen während der Corona-Pandemie wurde zwischen dem 29. Mai und dem 22. Juni 2020 in Großbritannien eine Online-Umfrage zu hypothetischen Triage-Dilemmata durchgeführt [27]. Den 763 Teilnehmern, die für die allgemeine Bevölkerung Großbritanniens repräsentativ waren, wurden Szenarien präsentiert, in denen ein einziges Beatmungsgerät zur Verfügung steht und 2 Patienten auf der Intensivstation aufgenommen und beatmet werden müssen. Die Patienten unterschieden sich in einer der folgenden Hinsichten: Überlebenschance, Lebenserwartung, Alter, erwartete Behandlungsdauer, Behinderung und Grad der Gebrechlichkeit. Die Teilnehmer sollten sich entweder für einen der beiden Patienten entscheiden oder konnten den Vorschlag machen, eine Münze zu werfen, um zu entscheiden. Die Mehrheit der Befragten bevorzugte Patienten, die eine höhere Überlebenschance (72\%-93\%), eine längere Lebenserwartung (78\%-83\%) oder eine kürzere Behandlungsdauer ( $88 \%-94 \%$ ) hatten, jünger (71\%-79\%) waren oder einen geringeren Grad an Gebrechlichkeit (60\%-69\%, 
alle $\mathrm{p}<0,001)$ aufwiesen. ${ }^{13}$ Wenn der Unterschied zwischen 2 Patienten klein war, entschied sich ein größerer Anteil dafür, die Entscheidung dem Zufall zu überlassen. Eine Mehrheit (58\%-86\%) war bereit, die Behandlung eines Patienten auf der Intensivstation abzubrechen, der eine geringere Überlebenschance hatte als ein anderer Patient, der sich gerade mit COVID-19 vorstellte. Die Befragten gaben auch an, dass sie bereit wären, medizinischem Personal und Patienten mit kleinen Kindern höhere Priorität einzuräumen. Wie sich zeigte, unterstützt die britische Öffentlichkeit einen weitgehend utilitaristischen Ansatz bei der Triage auf der Intensivstation angesichts einer überwältigenden Notlage. Interessanterweise befürworteten die Teilnehmer nicht nur Faktoren, die in den Triage-Leitlinien enthalten sind. Eine ähnliche in den USA durchgeführte Studie an 586 Teilnehmern kam zu etwa dem gleichen Ergebnis [13]. Insbesondere sind sich die Menschen darin einig, dass jüngeren Menschen eine größere Priorität zukommt als älteren. Da beide Studien aus dem angloamerikanischen Raum stammen, lassen sich ihre Ergebnisse wahrscheinlich nicht global verallgemeinern.

Halten wir fest: Es gibt mittlerweile große empirische Datensätze dazu, wie sich Menschen moralisch entscheiden. Hierbei zeigen sich kulturelle Unterschiede, die in der Diskussion über die Frage des Impfens gegen COVID-19 eine Rolle spielen müssen, weil es sich einerseits um eine globale Pandemie handelt, die Strategie des Impfens aber andererseits nicht von einem Land auf das andere übertragen werden kann.

\section{Diskussion}

Solange die Impfstoffe gegen SARS-CoV-2 nicht allen Impfwilligen zur Verfügung gestellt werden können, es sich also um knappe Güter handelt, stellt sich die Frage nach der Priorisierung. Wenn man diese vornimmt, geht es prinzipiell um die Optimierung einer Variablen (Anzahl der Toten, Lebensjahre oder QALYs), über die Einigkeit herrschen muss, weil bei jeder Opti-

13 In den Klammern wird jeweils ein Intervall angegeben, weil die Prozentsätze in Anhängigkeit von der Größe des Unterschieds im Einzelfall abhingen. mierung grundsätzlich vorher geklärt sein muss, was optimiert werden soll. Empirisch wurde festgestellt, dass die Auswahl der Variablen vom Infektionsgeschehen abhängen kann. Weil Priorisierung eine Form von Bewertung ist und die dabei abzuwägenden Werte sowohl intrinsisch als auch extrinsisch bzw. instrumentell sind und der Zweckrationalität unterliegen, lassen sich die Grundlagen moralischer Entscheidungen ebenfalls empirisch untersuchen. Die Entscheidungen selbst sind ethische, denn wenn man über sie gründlich genug nachdenkt, dann denkt man über die allgemeinen Grundlagen moralischer Entscheidungen - also über Ethik ${ }^{14}$ - nach.

Dennoch ist auch die Frage, ob man überhaupt priorisieren soll, nicht rein ethischer Natur, sondern hat ebenfalls empirische Aspekte. So lässt sich anhand von Rechenmodellen zeigen, dass eine schnelle Impfung zur bestmöglichen Abwendung von Tod und Leid die bessere Alternative zu einer langsameren, aber dafür entscheidungsoptimierten Impfstrategie ist: „Eine schnell umgesetzte, ungezielte Kampagne ist weitaus erfolgreicher als eine langsame, aber optimal gezielte Kampagne“15, schreiben hierzu Modellierer in einer noch nicht vom Review-Prozess bewerteten Arbeit [18]. Man kann ergänzen, dass in der Veterinärmedizin schon lange gilt, dass bei der Bekämpfung einer Epidemie „Schnelligkeit vor Genauigkeit" geht.

Letzteres scheint jedoch derzeit (Stand: 1.3.2021) vorzuliegen, denn Deutschland liegt beim Impfen im europaweiten Vergleich weit hinten ( $>$ Abb. 5). Insbesondere im Hinblick auf die Impfung mit dem Impfstoff von AstraZeneca ist bekannt, dass viele Menschen ihn ablehnen, er aber zugleich wirksam und in großer Zahl an Impfdosen vorhanden ist. Seine Verimpfung verläuft schleppend, was insbesondere angesichts einer aufgrund mutierter Varianten

14 Obwohl Moral und Ethik nicht selten verwechselt werden (in „Ethikkommissionen“ geht es nicht um Begründungsformen oder -prinzipien moralischer Entscheidungen, sondern nur um moralische Abwägungen), sind sie ebenso wenig dasselbe wie Rechnen und Mathematik.

15, „.... ] a rapidly deployed untargeted campaign is far more successful than a slow but optimally targeted one."

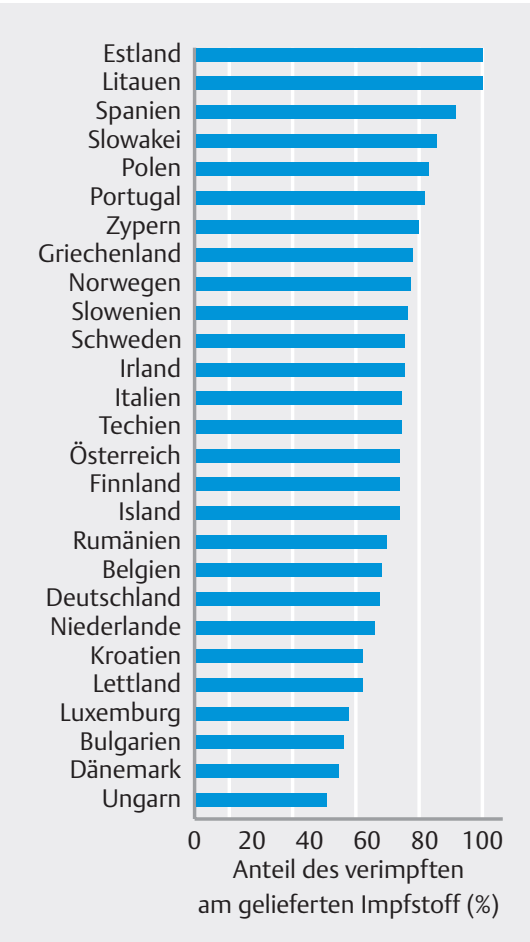

Abb. 5 Anteil des verimpften COVID-19 Impfstoffs (in Prozent) am gelieferten COVID-19-Impfstoff (info.Bild.de; Quelle: ECDC, BMG; Stand 25.2.2021)

des Virus drohenden (und beginnenden) „dritten Welle“ als besonders dramatisch erscheint. Denn zwischen den Impfwilligen und deren Impfung steht die Impfverordnung mit ihrer Priorisierung. Impfärzten könnten Strafen drohen (bis zu 25000 Euro werden politisch diskutiert [1]), wenn sie von der Priorisierung abweichen, Impfwillige, die noch nicht „dran“ sind, werden nicht geimpft, und in den Impfzentren herrscht gähnende Leere, weil viele, die den Impfstoff bekommen dürfen, keinen Termin vereinbaren oder den vereinbarten Termin zur Impfung mit dem genannten Impfstoff nicht wahrnehmen. Aus diesen Tatsachen folgt, dass man aus ethischen Gründen die derzeitige Priorisierung mindestens vorübergehend außer Kraft setzen müsste. Hunderttausende deutscher Ärzte stehen - sofern und sobald ihnen all dies klar geworden ist - vor dem zusätzlichen Dilemma, dass sie aus ärztlicher Sicht (Hippokrates: soviel wie möglich helfen und so wenig wie möglich schaden) der Verordnung nicht folgen sollten. Als Beamter des Landes Baden-Württemberg, der einen Eid geleistet hat, dem Land zu dienen, steht man zusätzlich vor dem Dilemma, ob mit „Land“ des- 
sen Verordnungen oder dessen Bürger gemeint sind.

Nur wenn jetzt innerhalb kürzester Zeit (3-4 Wochen) alle geimpft würden (und damit die dritte Welle möglicherweise verhindert würde), machte die Priorisierung weiterhin Sinn. Solange jedoch die Priorisierung das Impfen behindert, sollte sie ausgesetzt werden. Dass uns dies weiteres Kopfzerbrechen ersparen könnte, lässt sich an folgendem empirischen Sachverhalt beispielhaft erläutern: Bei der erwähnten Verweildauer im Pflegeheim gibt es Geschlechterunterschiede: Nach 2 Jahren sind schon 52 \% der Männer, aber erst 38 \% der Frauen verstorben [22]. Um hier Gerechtigkeit zu schaffen, müssten deutlich mehr als die Hälfte der Männer und entsprechend weniger Frauen geimpft werden. Um wieviel man hierbei von der Parität (50\%) abweicht, hängt davon ab, welche Variable optimiert wird (Tote, YLL oder QALY). Dies wäre dann wieder eine ethische Entscheidung - ebenso wie die, ob man diese Art der Geschlechtergerechtigkeit überhaupt will. Schenkt man den neuesten Modellierungen des Pandemieverlaufs Glauben $[20,21]$, dann werden sich die bisherigen Impfungen im Sinne einer geringeren $\mathrm{CO}$ VID-19-Infektionsrate in den geimpften Altersgruppen dahingehend auswirken, dass vor allem jüngere Menschen erkranken und zum Teil erhebliche Einbußen ihrer Lebensqualität über viele Jahre bei chronischen Verläufen („Long COVID“; [6]) erleiden.

Halten wir fest: Die Priorisierung der Impfung wirft komplexe Fragen auf, die empirischer, moralischer und ethischer Natur sind. Die Praxis der Priorisierung vor dem Hintergrund sich ändernder Rahmenbedingungen sollte dynamisch immer wieder neu abgewogen werden.

\section{Epilog anlässlich der Fahnenkorrektur}

Der Text dieses Beitrags wurde im Februar verfasst, ging Anfang März in den Satz und wurde in den Fahnen aktualisiert. In der zweiten und dritten Märzwoche erreichte das Problem der Impfpriorisierung aufgrund zweier Vorkommnisse eine vom Autor nicht vorhergesehene Konkretisierung und Aktualität, was das folgende kleine Nachwort rechtfertigen mag. Am 11.3. wurde von Gesundheitsminister Jens Spahn eine neue Impfverordnung - mit Gültigkeit ab dem 8.3. und publiziert am 11.3. - erlassen, in der auf 10 dicht beschriebenen Seiten nochmals eine überarbeitete Priorisierung der Impflinge in solche mit „höchster Priorität“ (Personen über 80, medizinisches Personal im Kontakt mit Corona-Patienten und hohem Expositionsrisiko etc.), „hoher Priorität“ (über 70, Vorliegen bestimmter Vorerkrankungen, medizinisches Personal mit erhöhtem Expositionsrisiko etc., Erzieher, Lehrer in Grund-, Sonder- und Förderschulen) und „erhöhter Priorität“ (über 60 Jahre, bestimmte Vorerkrankungen, Polizei, Feuerwehr, Lehrer, Lebensmitteleinzelhandel) erfolgte. Neu und besonders kontrovers war die Aufnahme der Erzieher und Lehrer, denn diese wurde mit der Notwendigkeit zur Aufrechterhaltung des Betriebs in Kitas und Schulen und damit zum Schutz des hierzu gebrauchten Personals begründet. Damit war ein neuer Gesichtspunkt neben der Minimierung der Toten und der Patienten auf Intensivstationen auch noch die Aufrechterhaltung des Präsenzbetriebs von Bildungseinrichtungen - in die Abwägungen aufgenommen. Aus meiner Sicht zu Recht [24]. In dieser Verordnung wurde jedoch zugleich festgestellt, dass unter bestimmten Bedingungen von der Priorisierung abgewichen werden kann, „wenn dies für eine effiziente Organisation der Schutzimpfungen oder eine zeitnahe Verwendung vorhandener Impfstoffe notwendig ist, “ also der Impfstoff sonst weggeworfen werden muss. „Von der Reihenfolge [...] kann zudem abgewichen werden, um eine dynamische Ausbreitung des Coronavirus SARS-CoV-2 aus hochbelasteten Grenzregionen (Ringimpfung) sowie in oder aus Hochinzidenzgebieten in der Bundesrepublik Deutschland (Riegelimpfung) zu verhindern“ [34]. Man hat hier also versucht, die Impfpriorisierung weiter zu differenzieren und den Gegebenheiten anzupassen, so wurde zugleich überlegt, dass die Priorisierung auch ausgesetzt werden kann bzw. muss, wenn es die Infektionsdynamik erfordert.

Ein konkreter Anwendungsfall für das Aussetzen der Priorisierung lag zum Zeitpunkt der Veröffentlichung der neuen Impfverordnung bereits vor: Angesichts hoher Infektionszahlen war am 9.3.2021 von der sächsischen Gesundheitsministerin Petra
Köpping im Rahmen einer Kabinettssitzung entschieden worden, dass sich im Vogtland-Kreis alle Einwohner ab 18 Jahren gegen das Coronavirus impfen lassen können, weil die 7-Tage-Inzidenz in diesem Landkreis an diesem Tag nach Angaben des Robert-Koch-Instituts bei 251 lag [32]. Am 15.3. forderten die Stadträte der Stadt Hof angesichts einer 7-Tage-Inzidenz von 290 [33] ebenfalls ein Aussetzen der Impfpriorisierung. Es hatte sich nämlich gezeigt, dass zwischen den Impfwilligen einerseits und dem reichlich vorhandenen Impfstoff von AstraZeneca andererseits die Impfverordnung mit ihrer seitenlangen Liste unterschiedlicher Impfprioritäten stand. Wenn man alles ganz genau und ganz gerecht machen will, dann geht es nicht schnell. Und schnell gehen muss die Abhilfe, wenn „Landunter“ ist.

Das zweite Vorkommnis betraf den Impfstoff der Firma AstraZeneca, von dem am 12.3. nach einer Meldung der Nachrichtenagentur Reuters zu Angaben der WHO weltweit bereits mehr als 268 Millionen Dosen Impfstoff verimpft worden waren, ohne dass dies auch nur einen einzigen Todesfall verursacht hätte [44]. In Deutschland hingegen entwickelten etwa Mitte März 6 Frauen und ein Mann im Alter zwischen 20 und 50 Jahren Sinusvenenthrombosen, jeweils 4 bis 16 Tage nach der AstraZeneca-Impfung. 3 Personen verstarben. Auch in den umliegenden Ländern wie Dänemark, Frankreich oder Italien waren solche Fälle beobachtet worden, jeweils auch nur sehr wenige. In den Hirnvenen bilden sich über Tage hinweg Gerinnsel, die den Blutabfluss behindern. Im Gehirn steigt der Druck, es kann zu Blutungen kommen, zu Hirnschäden und zum Tod. Dieses Krankheitsgeschehen ist sehr selten: Von einer Million Menschen kommt es etwa bei 15 Personen innerhalb eines Jahres dazu - ohne Impfung. Betroffen sind eher Frauen als Männer, eher Junge als Alte.

Das Paul-Ehrlich-Institut (PEI) bezeichntete die Fälle als ungewöhnliche Häufung, wertete sie als Signal für ein erhöhtes Impfrisiko und empfahl daher das Aussetzen der Impfung mit diesem Impfstoff. Angesichts der bereits aus diesem Grund erfolgten Aussetzung des Impfens mit dem Impfstoff von AstraZeneca in der Mehrzahl der Länder der EU ( $\triangleright$ Abb. 6) und der Empfehlung des PEI konnte Gesundheitsminis- 


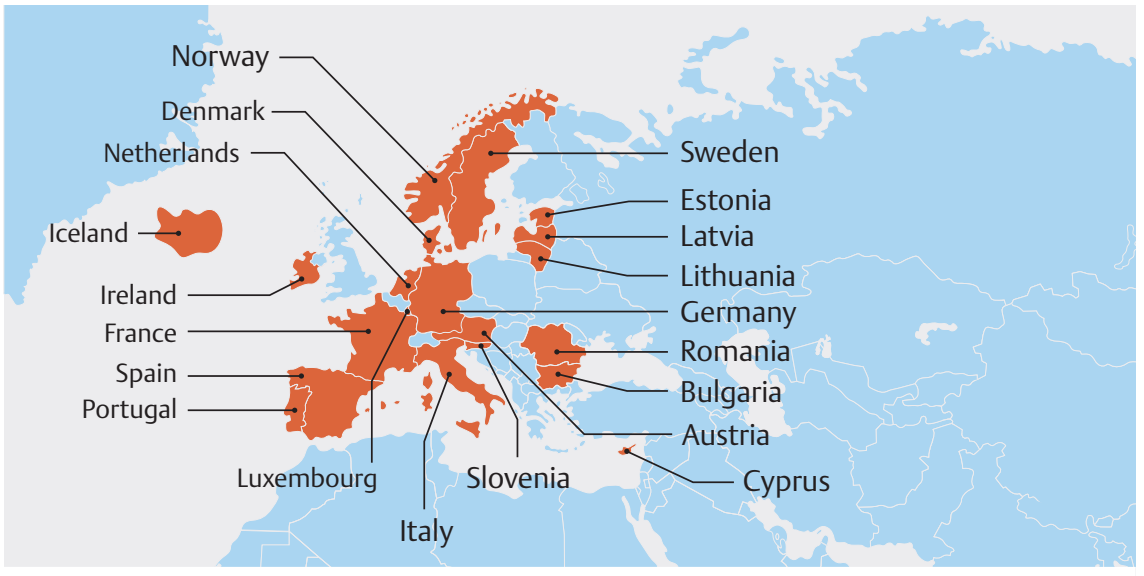

- Abb 6 Karte mit den Ländern (rot markiert), die einen Impfstopp im Hinblick auf den Impfstoff von AstraZeneca am 15.3. verhängt hatten (nach Daten aus [33]).

ter sich schwerlich den Nachbarn und der Wissenschaft widersetzen. Er verkündete daher am Nachmittag des 15.3. (Montag) mit sofortiger Wirkung das Aussetzen der Impfung mit diesem Impfstoff auch in Deutschland: Impfzentren brachen die Arbeit sofort ab, willige Impflinge wurden nach Hause geschickt und der bereits vorbereitete Impfstoff weggeworfen. Am gleichen Abend meldet die Leiterin der Europäischen Arzneimittelagentur (EMA), dass es keinen Hinweis für einen kausalen Zusammenhang zwischen Impfung und Gehirnvenenthrombosen gebe. Man werde dies jedoch weiter untersuchen. Am Nachmittag des 18.3. (Donnerstag) gegen 17 Uhr hat die EMA den AstraZeneca-Impfstoff für unbedenklich erklärt, sodass er ab sofort wieder verimpft werden konnte. Der Impfstoff wird nun mit einem Warnhinweis versehen und der Aufklärungsbogen aktualisiert.

Diese Episode in der deutschen Pandemie-Geschichte ist in mehrfacher Hinsicht bemerkenswert, denn es ging erstens um ein fragwürdiges Signal, auf das zweitens eine noch fragwürdigere Reaktion erfolgte, die drittens zu einigen hundert Toten innerhalb der nächsten Monate führen wird - was jeder in 2 Minuten auf der Rückseite eines Bierdeckels berechnen kann.

Ad 1) Wie schon erwähnt, handelte es sich bei in 6 der 7 Fälle um Frauen mit Sinusvenenthrombose (mittlerweile sind es in Deutschland 13 Fälle, 12 davon Frauen). Sie waren jüngeren oder mittleren Alters und 5 von ihnen hatten ein Kontrazeptivum (Pille) eingenommen. Man weiß, dass dies mit einem erhöhten Thromboserisiko ver- bunden ist ${ }^{16}$. Da in den letzten Wochen vor allem jüngere Frauen (Erzieherinnen und Lehrerinnen) mit dem Impfstoff von AstraZeneca geimpft wurden, waren sie in der Gruppe der Geimpften überrepräsentiert. Man kann leicht ausrechnen, dass dies bei mehr als einer Million Impfungen mit dem Impfstoff von Astra-Zeneca zu einem zufälligen Auftreten von Gehirnvenenthrombosen innerhalb des bisherigen Impfzeitraums in 3 bis 5 Fällen führen kann. Bei Männern - einer ist statistisch gesehen keiner - gab es also gar kein Risikosignal, bei älteren Frauen auch nicht ${ }^{17}$. Und ob es bei jüngeren Frauen wirklich eines gab, wird die Zukunft sicherlich zeigen (weltweit werden Milliarden von Menschen geimpft).

Ad 2) Wenn dies so ist, dann gab es nie auch am Montag den 15.3. nicht - einen Grund, das Impfen mit dem Impfstoff von

16 In einer Stellungnahme der Gesellschaft für Thrombose- und Hämostaseforschung (GTH) vom 19.3.2021 geht man von einer immunologischen Genese der Sinus-/ Hirnvenenthrombosen aus: „Ein wichtiger Pathomechanismus wurde mittlerweile innerhalb der GTH unter Führung der Greifswalder Arbeitsgruppe um Andreas Greinacher aufgeklärt. Durch die Impfung kommt es wahrscheinlich im Rahmen der inflammatorischen Reaktion und Immunstimulation zu einer Antikörperbildung gegen Plättchenantigene. Diese Antikörper induzieren dann [...] eine massive Thrombozytenaktivierung. [...] Dieser Mechanismus [...] konnte bei 4 Patienten mit einer Sinus-/Hirnvenenthrombose nach Impfung mit dem AstraZeneca COVID-19 Vakzin [...] nachgewiesen werden“ [47].

17 In Frankreich wurde daher das Impfen auf Frauen über 55 Jahren beschränkt.
AstraZeneca auszusetzen. Nur ältere Frauen über 55 oder nur Männer zu impfen, hätte genügt. Mit dieser sehr einfachen Priorisierung - „Impft doch bitte die nächsten paar Tage bis auf Widerruf nur Männer“ wäre es also getan gewesen. Diese Lösung hätte zudem den Charme gehabt, dass sie ein bisschen mehr Impfgerechtigkeit hergestellt hätte. Denn bislang impfen wir ja die Älteren, weil sie ein höheres Erkrankungsrisiko haben. Dies haben aber auch die Männer, denn „männliches Geschlecht“ ist nach „höherem Alter“ der zweitgrößte Risikofaktor für eine Coronainfektion. Die vorgeschlagene sehr einfach durchzuführende Priorisierung hätte also sogar etwas mehr Impfgerechtigkeit erzeugt. Von Dienstag bis Freitag wären täglich etwa 100000 Männer mit dem Impfstoff von AstraZeneca geimpft worden, nichts hätte angehalten werden müssen, nur die Hälfte aller Termine hätte abgesagt und neue vereinbart werden müssen. Die Impfmaschinerie wäre weitergelaufen.

Ad 3) Die etwa 400000 Nichtgeimpften schleppen wir bis zum Ende des Impfens mit: Wieviel wir auch immer Impfen, es werden zu einem gegebenen Zeitpunkt bis zum Ende der Impfungen gegen Corona immer 400000 Menschen weniger geimpft sein. Bei einer Inzidenz von 100 pro 100000 pro Woche sind das 400 Infizierte pro Woche mehr oder etwa 60 pro Tag. Wenn die Kanzlerin Wort hält, dann erhalten bis Ende September alle Impfwilligen eine Impfung. Von Mitte März bis Ende September sind es etwa 200 Tage, was bei 60 Infizierten/Tag in 12000 zusätzlichen Infizierten resultiert. Bei einer Sterbewahrscheinlichkeit von 3\% sind das 360 zusätzliche Todesfälle. Sofern die Geschwindigkeit des Impfens sich nicht stärker erhöht als von der Kanzlerin angenommen und sofern nicht irgendeine andere Therapie, die sicher vor dem Tod schützt, entwickelt wird, ist das die Größenordnung des Schadens durch die 4 Tage Impfpause, die man sich durch eine einfache Impfpriorisierung hätte ersparen können. Warum weder am PEI noch im Gesundheitsministerium irgendeiner der jeweils Hunderten dortigen hochbezahlten Experten diese Überlegungen angestellt und entsprechendes Handeln vorgeschlagen hat, weiß ich nicht. Aber es macht mir Angst. Zwischen Montag (15.3.) und Donnerstag (18.3.) wurden keine neuen Daten 
erhoben oder ausgewertet (so schnell geht das nicht). Es wurde also nur 4 Tagen das gedacht, was man auch in 2 Minuten denken konnte - sogar schon vor dem 15.3.

Die Priorisierung von Menschen bei Impfungen zum Erreichen des bestmöglichen Ergebnisses für Alle ist eine hohe Kunst.

Anmerkung bei der zweiten Fahnenkorrektur: Am Abend des 30.3. beschlossen die Gesundheitsminister von Bund und Ländern aufgrund weiterer Fälle von Hirnvenenthrombosen, dass der Impfstoff von AstraZeneca in der Regel nur noch bei Menschen ab 60 Jahren eingesetzt werden soll, wonach in einer anschließenden Schaltkonferenz von Kanzlerin Angela Merkel mit Bundesgesundheitsminister Jens Spahn und den Ministerpräsidenten gemeinsame Änderungen der Impfkampagne angekündigt wurden. Grundlage der Entscheidung war eine Empfehlung der Ständigen Impfkommission (STIKO). Da in Deutschland derzeit Millionen von Menschen über 60 noch nicht geimpft sind, für die das Impfrisiko nach den vorhandenen Daten nicht gestiegen ist, kann der vorhandene AstraZeneca-Impfstoff weiter Teil der Impfkampagne sein. „Durch die aktualisierte Empfehlung gib es in der Summe keine einzige Impfdosis weniger, es geht nur um Umschichtung derselben Impfstoffdosen. Der Fortgang der Impfkampagne muss nach unserer Modellierung nicht wesentlich verzögert sein“, antwortete mir Prof. Thomas Mertens (bevor er Chef der STIKO wurde, war er mein Kollege an der Ulmer Fakultät) auf eine Anfrage, die ich 5 Minuten vor Mitternacht am 30.3. per E-Mail an ihn gerichtet hatte, prompt 20 Minuten später. Da der wahrscheinliche Pathomechanismus der Hirnvenenthrombosen Männer nicht ausschließe und bislang ganz überwiegend junge Frauen - Erzieherinnen und Lehrerinnen - geimpft wurden, können die STIKO über das tatsächliche Risiko der Männer nichts genaues sagen. Die STIKO ist also vorsichtig - und das ist gut so. Zugleich muss das Impfen nicht angehalten werden. Und das ist die wichtigste Nachricht.

Interessenkonflikt

Es liegt kein Interessenkonflikt vor.
Korrespondenzadresse

Prof. Dr. Dr. Manfred Spitzer

Universität Ulm

Abteilung für Psychiatrie

Leimgrubenweg 12-14

89075 Ulm, Deutschland

Literatur

[1] Anonymus. Impfdrängler: Koalition plant bis zu 25000 Euro Strafe. Augsburger Allgemein 25.2.2021 https://www. augsburger-allgemeine.de/politik/Impfdraengler-Koalition-plant-bis-zu-25-000Euro-Strafe-id59192396.html; abgerufen am 2.3.2020

[2] Archer SL. Providing care for the $99.9 \%$ during the COVID-19 pandemic: How ethics, equity, epidemiology, and cost per QALY inform healthcare policy. Healthc Manage Forum 2020; 33: 239-242

[3] Arolas HPI, Acosta E, López-Casasnovas G et al. Years of life lost to COVID-19 in 81 countries. Sci Rep 2021; 11: 3504

[4] Bambery B, Douglas T, Selgelid MJ et al. Influenza Vaccination Strategies Should Target Children. Public Health Ethics 2018; 11: 221-234

[5] Briggs AH, Goldstein DA, Kirwin E et al. Estimating (quality-adjusted) life-year losses associated with deaths: With application to COVID-19. Health Econ 2018; 30: 699-707

[6] Buonsenso D, Munblit D, De Rose C et al. Preliminary Evidence on Long COVID in children. medRxiv 2021. doi. org/10.1101/2021.01.23.21250375

[7] Campos MR, Schramm JMA, Emmerick ICM et al. Burden of disease from COVID-19 and its acute and chronic complications: reflections on measurement (DALYs) and prospects for the Brazilian Unified National Health Syste. Cad Saude Publica 2020; 36 (11): e00148920

[8] Creech CB, Walker SC, Samuels RJ. SARSCoV-2 Vaccines. JAMA 2021. doi:10.1001/ jama.2021.3199

[9] Giubilini A, Savulescu J, Wilkinson D. COVID-19 vaccine: vaccinate the young to protect the old? J Law Biosci 2020; 7: Isaa050

[10] Giubilini A, Savulescu J, Wilkinson D. Queue questions Ethics of COVID-19 vaccine prioritization. Bioethics 2021. doi: 10.1111/ bioe. 12858

[11] Hall J, Viney R. Quality adjusted life years in the time of COVID-19. Aust Health Rev 2021; 45: 12-13

[12] Halpern SD, Truog RD, Miller FG. Cognitive Bias and Public Health Policy During the COVID-19 Pandemic. JAMA 2020; 324: 337-338

[13] Huseynov S, Palma MA, Nayga RM. General Public Preferences for Allocating Scarce
Medical Resources During COVID-19. Front Public Health 2020; 8: 587423

[14] Jacobs K et al (Hrsg.). Pflege-Report 2016. „Die Pflegenden im Fokus“. Stuttgart: Schattauer; 2016

[15] Kohli M, Maschio M, Becker D et al. The potential public health and economic value of a hypothetical COVID-19 vaccine in the United States: Use of cost-effectiveness modeling to inform vaccination prioritization. Vaccine 2021; 39: 1157-1164

[16] Meißner M. Was ist ein Qaly? Dtsch Arztebl 2010; 107: A-546/B-476/C-468

[17] Miles DK, Stedman M, Heald AH. Stay at Home, Protect the National Health Service, Save Lives": A cost benefit analysis of the lockdown in the United Kingdom. Int J Clin Pract 2020; 13: e13674

[18] Moore S, Hill EM, Dyson L et al. Modelling optimal vaccination strateg y for SARSCoV-2 in the UK. medRxiv 2020. doi. org/10.1101/2020.09.22.20194183

[19] Reddy R. Population health, economics and ethics in the age of COVID-19. BMJ Global Health 2020; 5: e003259

[20] Schuppert A, Weber-Carstens S, Karagiannidis C. Simulation der Intensivbettenauslastung für COVID-19 in Abhängigkeit von der Infektionsdynamik und dem zu erwartenden Impfeffekt 2021

[21] Schuppert A, Theisen S, Fränkel P et al. Bundesweites Belastungsmodell für Intensivstationen durch COVID-19. Med Klin Intensivmed Notfmed 2021. doi. org/10.1007/s00063-021-00791-7

[22] Schwinger $A$, Jürchott $K$, Tsiasioti $C$ et al. Pflegebedürftigkeit in Deutschland. 2016

[23] Shah SMA, Mohammad D, Qureshi MFH et al. Prevalence, Psychological Responses and Associated Correlates of Depression, Anxiety and Stress in a Global Population, During the Coronavirus Disease (COVID-19) Pandemic. Community Ment Health J 2021; 57: $101-110$

[24] Spitzer M. Öffnet die Schulen. Nervenheilkunde 2021; 40: 296-311

[25] Torales J, O’Higgins M, Castaldelli-Maia JM et al. The outbreak of COVID-19 coronavirus and its impact on globalmental health. Int J Soc Psychiatry 2020; 66: 317-320

[26] Weekes M, Jones NK, Rivett L et al. Single-dose BNT162b2 vaccine protects against asymptomatic SARS-CoV-2 infection. Authorea 2021. doi:10.22541/ au.161420511.12987747/v1

[27] Wilkinson D, Zohny H, Kappes A et al. Which factors should be included in triage? An online survey of the attitudes of the UK general public to pandemic triage dilemmas. BMJ Open 2020; 10: e045593

[28] Wößmann L. Folgekosten ausbleibenden Lernens: was wir über die coronabedingten Schulschließungen aus der Forschung lernen können. Ifo Schnelldienst 2020; 73: 38-44

[29] Wu B. Social isolation and loneliness among older adults in the context of COVID-19: 
a global challenge. Glob Health Res Policy 2020; 5: 27

[30] Raftery R. Who should be first in line for a covid-19 vaccine?Assessing effectiveness and cost effectiveness. BMJ, Blog, 17.11.2020 https://blogs.bmj.c 2020; $\mathrm{m} /$ bmj/2020/11/17/james-raftery-whoshould-be-first-in-line-for-a-covid-19-vaccine-assessing-effectiveness-and-cost-effectiveness/; abgerufen am 28.1.2021

[31] Awad E, Dsouza S, Kim R et al. The Moral Machine experiment. Nature 2018; 563: 59-64

[32] Anonymus Impfangebot für alle ab 18 Jahren im Vogtland. MDR 9.3.2021, 18.40 Uhr https://www.mdr.de/nachrichten/ sachsen/chemnitz/vogtland/corona-impfen-ab-achtzehn-100.html; abgerufen am 18.3.2021

[33] Anonymus. Hof: Stadträte fordern Abkehr von der Impf-Priorisierung. BR24 15.3.2021, 13.44 Uhr (https://www. br.de/nachrichten/bayern/hof-stadtraetefordern-abkehr-von-der-impf-priorisierung, SRj0mks; abgerufen am 19.3.2021

[34] Bundesministerium für Gesundheit, BMG (2021) Verordnung zum Anspruch auf Schutzimpfung gegen das Coronavirus SARS-CoV-2 (Coronavirus-Impfverordnung - CoronalmpfV) vom 10. März 2021. Bundesanzeiger herausgegeben vom Bundesministerium für Justiz und für Verbraucherschutz. BAnz AT 11.03.2021 V1

[35] Campos MR, Schramm JMA, Emmerick ICM et al. Burden of disease from COVID-19 and its acute and chronic complications: reflections on measurement (DALYs) and prospects for the Brazilian Unified National Health System. Cad Saude Publica 2020; 36(11): e00148920
[36] Feng X, Kim DD, Cohen JT et al. Using QALYsversus DALYs to measure cost-effectiveness:How much does it matter? International Journal of Technology Assessment in Health Care 2020; 36: 96-103

[37] Gold MR, Stevenson D, Fryback DG. HALYs and QALYs and DALYs, Oh My: Similarities and Differences in Summary Measures of Population Health. Annu Rev Public Health 2002; 23: 115-134

[38] Bubar KM, Reinholt K, Kissler SM et al. Model-informed COVID-19 vaccine prioritization strategies by age and serostatus. Science 2021; 371: 916-921

[39] Medlock J, Galvani AP. Optimizing influenza vaccine distribution. Science 2009; 325: 1705-1708

[40] Buss LF, Prete CA Jr, Abrahim CMM et al. Three-quarters attack rate of SARS-CoV-2 in the Brazilian Amazon during a largely unmitigated epidemic. Science 2021; 371: 288-292

[41] Logue JK, Franko NM, McCulloch DJ et al. Sequelae in adults at 6 months after COVID-19 infection. JAMA Netw Open 2018; 4: e210830

[42] Hansen CH, Michlmayr D, Gubbels SM et al. Assessment of protection against reinfection with SARS-CoV-2 among 4 million PCR-tested individuals in Denmark in 2020: a population-level observational study. The Lancet 2021. doi.org/10.1016/S01406736(21)00575-4

[43] Khamsi R. If a coronavirus vaccine arrives, can the world make enough? Nature 2020; 580: 578-580

[44] Farge E. WHO says its advisory committee looking at AstraZeneca vaccine issues. Reuters, 12.3.2021 https://www.reuters. com/article/health-coronavirus-who-vac cines/update-2-who-says-its-advisorycommittee-looking-at-astrazeneca-vac cine-issues-idUSL8N2LA34M; abgerufen am 12.3.2021

[45] Ftzpatrick MC, Galvani AP. Optimizing age-specific vaccination. Vaccination strategies are not one-size-fits-all. Science 2021; 371: 890-892

[46] Anonymus. FEARS around blood clot links with the AstraZeneca Covid vaccine have sent Europe into a panic. The Sun, 16.3.2021 https://www.thesun.co.uk/ news/14350112/boris-johnson-oxford-as trazeneca-jab-safe-eu-ban/; abgerufen am 16.3.2021

[47] Oldenburg J, Klamroth R, Langer F et al. Aktualisierte Stellungnahme der GTH nach dem Beschluss der EMA, die Impfungen mit dem AstraZeneca COVID-19 Vakzin fortzusetzen. Gesellschaft für Thrombose- und Hämostaseforschung, 19.3.2021 (https://gth-online. org/wp-content/uploads/2021/03/GTH_Stellungnahme_AstraZeneca_3_19032021-3. pdf; abgerufen am 22.3.2021

\section{Bibliografie}

DOI https://doi.org/10.1055/a-1298-1189 Nervenheilkunde 2021; 40: 367-376 (c) 2021. Thieme. All rights reserved. Georg Thieme Verlag KG, Rüdigerstraße 14, 70469 Stuttgart, Germany ISSN 0722-1541 